\title{
A Secondary Data Analysis of a Youth Yoga Program
}

\author{
Brandon Eggleston PhD MPH MCHES CPH \\ Associate Professor of Community Health, Lead Faculty BSPH Program (online), \\ National University-San Diego, Cailfornia \\ beggleston@nu.edu
}

\begin{abstract}
:
Background: Children experience today an unprecedented amount of stress and anxiety in their daily life. Yoga is one potential activity that can may help children to improve mindfulness which has been shown to be positively associated with greater levels of psychosocial, emotional and physical well-being. This study attempts to identify whether there is an association between doing yoga and well-being in $4^{\text {th }}$ and $5^{\text {th }}$ grade students who participated in a yoga intervention and then determine the statistical significance of this association while controlling for time (pre-and post-survey), yoga intervention (yes/no) and grade level of the student.

Methods: A secondary data analysis was done using a seventeen-question survey which was administered to $4^{\text {th }}$ and $5^{\text {th }}$ grade students in three elementary schools(January-March, 2014). The survey was administered to the students before and after the eight week yoga intervention and five outcome ordinal variables were compared over time. Data was analyzed using SPSS 22.0 and included both descriptive and inferential analyses.

Results: Non-parametric tests identified significant $(p<.05)$ changes over time for five well-being outcome measures: self-esteem, interpersonal relationships, somatization, concentration, and physical health.

Conclusion: Data support the hypothesis that there was a statistically significant relationship between practicing yoga and improvement of self-esteem, interpersonal relationships, and concentration. Further, more scientific research should be done to validate and confirm these findings using randomized, controlled trials if possible.
\end{abstract}

Keywords: Yoga, children, school health, mindfulness, well-being, mental health

\section{INTRODUCTION}

Well-being is not merely the absence of disease in the body, but encompasses all aspects of an individual including mental, social, and emotional health. Wellness is the opposite of illness and focuses on both the individual's body functioning at an optimal level and feeling good in the mind (perception/attitude). Well-being can be described in a variety of ways. Three examples of well-being definitions/descriptions include the following: 1) Having positive emotions and moods; 2) The absence of negative emotion; and, 3) Feeling satisfied and content. Well-being is something that can be validly measured and provide a meaningful scientific outcome in research. According to a longitudinal experimental research done by the CDC, well-being is associated with self-perceived health, longevity, healthy behaviors, and productivity among others. From a health promotion standpoint, well-being empowers individuals to have control over their health (internal locus of control) which makes it easier to improve their health.

Studies have examined the multiple individual, social, and ecological determinants of well-being in populations. Generally, findings suggest that life satisfaction is dependent on the availability of basic needs (food, shelter, clothing, transportation, and healthcare) being met on a consistent basis. However, there were other determinants that were associated with well-being independent of basic needs such as genes, personality, age, gender, income, profession, marital status, and relationships. Many genetic and biologically related personality factors are considered by many biologists and psychologists to be independent of societal and environmental influences. Feelings of happiness and contentment can be inherited through genes (nature) and also directly influenced by the environment (nurture). As far as age and gender, men and women can have different levels and ideas about well-being and younger and older people differ also. Income has a direct influence on wellbeing up to a salary of $\$ 75,000$ annually. Above $\$ 75,000$, that income has no significant positive 
impact on well-being. The strongest predictor of well-being is supportive relationships (Well-being concepts, 2015).

\section{BURDEN OF THE DISEASE AND BEHAVIOR}

Children in the United States are suffering an unprecedented, and perhaps, previously unreported amount of anxiety, depression and emotional distress along with increasing rates of poor nutrition, obesity and incidence of adult-related, chronic diseases such as Type II Diabetes. Children, now more than ever, are suffering from adult maladies and are faced with coping with the challenges and stress at an earlier age. If these conditions are identified and intervened upon in childhood or adolescence, versus adulthood, the outcomes are substantially better.

Children can benefit from empowering lifestyles which lead to more self-confidence, lower anxiety levels, emotional stability and peaceful attitude. It is essential for children and adolescents to develop life skills that can enable them to take responsibility for their own lives respect themselves and others and increase their confidence in their potential.

Therapies and solutions for children include both preventative and treatment interventions.

Medications are routinely used to treat measures that are associated with well-being, such as anxiety and depression. However, According to the 2007 National Health Interview Survey (NHIS), which included questions on the use of Complementary and Alternative Medicine (CAM) by Americans, yoga is the sixth most commonly used CAM practice among adults. More than 13 million adults practiced yoga in the past year and a 2007 survey found that more than 1.5 million children practiced yoga in the past year.

Yoga is a mind and body practice which has origins in ancient Indian philosophy and dates back to 3,000 BCE. Yoga combines physical postures, breathing exercises, meditation and philosophy. There are six branches of yoga which include Bhakti, Karma, Raja, Tantra, Jnana, and Hatha. In the United States and Europe, Hatha Yoga which focuses on the physical practice of yoga, includes asanas (postures), pranayama (breathing exercises) and dhyana (meditation). There are also several styles of Hatha Yoga that are widely practiced such as Ananda, Anusara, Ashtanga, Bikram, Iyengar, Kripalu, Kundalini, and Viniyoga.

The physical postures and breathing work of yoga directs those who practice to be mindful and present. If one is focused on postures and breathing, that individual can focus on that moment and which can reduce anxiety by not focusing on the past or future.

\section{Purpose of The Study}

The purpose of this study was to examine the effect of a youth yoga program on measures of wellbeing for fourth and fifth grade students in San Diego County, California.

\section{LiMitATiONS}

There have been limited studies on the specific benefits of yoga for children. Many studies did not follow an experimental design, but were merely exploratory in nature. Studies in adults, while promising, may not be inferred to children entirely (Galatino, Galbavy, \& Quinn, 2008).

\section{LITERATURE REVIEW}

\subsection{Yoga for Children}

Studies of yoga in children are somewhat limited, but have increased in recent years. With the most serious limitation being lack of randomized controlled trials with high numbers of children/ adolescents. Yoga for children should be taught with the target audience in mind with regard to age and physical abilities as well as holding the interest of the young child or adolescent (Khalsa, HickeySchultz, Cohen, Steiner, \& Cope, 2012). Yoga is an acceptable and feasible activity in a school setting and has the potential of playing a protective or preventive role in maintaining mental health (Galatino, Galbavy, \& Quinn, 2008). Today's child is described as stressed out, undernourished and sedentary. As of 2008, studies of yoga in children were of varying quality and none were considered rigorous in regards to research design. Yoga is one mid-body therapy that has emerged as an educational based intervention and to improve various medical conditions for children. 


\subsection{Yoga in Schools}

Yoga is increasingly being used in classrooms across the United States as a way to enhance students behavioral and academic functioning (Frank, Bose, \& Schrobenhauser-Clonan, 2014). Studies were done in two elementary schools in Israel with children 8-12 years old using three questionnaires that incorporated self-assessment with parent and teacher assessment. This study was designed as a way to relieve stress in an area under bombardment. The results of the pre- and post-test intervention was that yoga may be beneficial for children in a postwar stress situation (An, Avshalon, \& Ehud, 2010).

In another study, participants included 49 students attending an alternative education school in an urban inner-city school district. The program was specifically designed for middle-high school-aged students (Frank, Bose, \& Schrobenhauser-Clonan, 2014). The study went beyond yoga due to the high-risk student population which included areas such as stress response, physical and emotional awareness, self-regulation and healthy relationships (Khalsa, Hickey-Schultz, Cohen, Steiner, \& Cope, 2012).

One of the most well-known yoga programs for children is the Yoga Ed program. This program was implemented in a number of secondary schools across the United States and includes simple yoga postures, breathing exercises, visualization and games with emphasis on fun and relaxation while minimizing risk and without unnecessarily complex, physically athletic or demanding activities. An important part of Yoga Ed is the development and training in the cognitive skills of mindfulness and self-awareness and incorporation of a yoga-based psychological and philosophical attitude. That being said, some schools have not or will not adopt a yoga program due to the perceived religiosity of yoga. Additionally, some studies have had participants opt out due to religious reasons.

Though Yoga Ed is one of the largest yoga programs in the United States, the Yoga Ed program, was studied on a normative population in a rural setting. When the population is considered normative, the benefits may not be as great given the baseline of the students (Khalsa, Hickey-Schultz, Cohen, Steiner, \& Cope, 2012).

\subsection{Yoga for Mental Health Issues}

Children and adolescents could benefit from learning to cope with stress. The skill of coping with stress is important for good mental health for all people. Learning this in childhood can help ensure a healthy transition into adulthood. Yoga and meditative processes can evoke a calming effect which can lead to a host of other benefits, both mentally and physically (Santangelo, 2012).

Yoga can help children learn to focus and be mindful which helps to reduce stress and tension. A study by Mendelson and colleagues (2010) found that elementary aged students in a high-poverty urban setting had less rumination (intrusive thoughts) after 12 weeks in a yoga program. This is significant because stress responses can progress into clinical anxiety and depression and adolescents are confronted with a variety of life lessons from both school and home. Unmanaged stress is now believed to be a contributing and/or causal factor in the development of mental health and behavioral problems that are prevalent in adolescents, including anxiety, depression, and behavioral problems (Khalsa, Hickey-Schultz, Cohen, Steiner, \& Cope, 2012).

A recent survey study indicated that $7.5 \%$ of adolescents meet the Diagnostic and Statistical Manual for Mental Disorders, Fourth Edition (DSM-IV) criteria for one or more mental health conditions. The age of onset of most of these disorders occurs in childhood and adolescence which places further emphasis on early intervention for children.

Transformative Life Skills (TLS) is a universal, classroom-based program for use with adolescents in middle or high school settings with high-risk students (Frank, Bose, \& Zelmer, Transformative life skills and training program curriculum, 2012). Students who participated in the Transformative Life Skills program demonstrated significant reductions in anxiety, depression and global psychological distress(Frank, Bose, \& Schrobenhauser-Clonan, 2014). In the TLS study, after adjusting p values for multiple comparisons, statistically significant and substantively meaningful improvements were found on measures of student anxiety, $\mathrm{t}(49)=-3.51, \mathrm{p}=.01, \mathrm{~d}=0.23$; depression, $\mathrm{t}(49)=-3.29, \mathrm{p}=.01, \mathrm{~d}=$ 0.32 ; and global symptoms index (GSI), $\mathrm{t}(49)=-2.69, \mathrm{p}=.01, \mathrm{~d}=0.40$. As this study was done on a small number of at-riskyouth with no control group, these results may not be generalizable.

A study by Shapiro et al. concludes that yoga appears to be a promising intervention for depression - 
it is cost-effective and easy to implement, producing many beneficial emotional, psychological and biological effects, as supported by observations in this study (Shapiro, Cook, Davydov, Ottaviani, \& Leuchter, 2007). Reductions in negative mood occurring from before to after yoga classes were greater for subjects scoring higher on scales of depression and anxiety than those scoring lower. Similar results were seen in an Israeli study with the majority of children in the yoga group reporting enhanced well-being, while children in an exercise group reported greater self-esteem (An, Avshalon, \& Ehud, 2010).

With regard to negative emotions, yoga participants in another study showed statistically significant differences over time relative to controls on measures of anger control and fatigue/inertia (Khalsa, Hickey-Schultz, Cohen, Steiner, \& Cope, 2012). Yoga and meditation have been shown to reduce perceived stress and improve mood and emotional disturbances (Nidhi, Padmalatha, Nagarathna, \& Amritanshu, 2012). The Veterans Health Administration (VA) and the US Department of Defense recommend Yoga as a potential adjunctive treatment to manage the hyperarousal symptoms of posttraumatic stress disorder (PTSD) (Skowronek, Mounsey, Handler, \& Guthmann, 2014).

Pharmaceuticals are most notably used to treat Attention-Deficit/hyperactivity disorder (ADHD), however, these medications are strong and powerful and not without risk. Jensen et al. unfortunately did not find that yoga significantly reduced the symptoms of ADHD, but holds that further research is warranted (Jensen \& Kenny, 2004).

Khalsa et al. studied yoga on performance anxiety in musicians and found that yoga and medications techniques showed promising results (Khalsa, Shorter, Cope, Wyshak, \& Sklar, 2009). This information could be translated to students who experience stress in school due to tests or presentations.

\subsection{Yoga for Specific Physical Health Issues}

Yoga has been shown to alleviate many specific physical health issues in adults and children. It is recommended that clinicians consider the addition of non-medical intervention when patients do not improve with medications and treatments as yoga was shown to be effective in reducing stress levels(Menon, Satyanand, \& Karishma, 2013). Studies have shown that savasana is as effective as electromyography (EMG) biofeedback in reduction of tension headache. Practice of savasana for three months has demonstrated an improvement of headache, insomnia, and nervousness in a study of 86 patients. The deep breathing of yoga exercises can provide extra oxygen to the blood and causes the body to release endorphins, which can reenergize and promote relaxation (An, Avshalon, \& Ehud, 2010).In a study of adolescents with inflammatory bowel disease, participants reported lower levels of functional disability, having lower scores for gastrointestinal symptoms and emotion-focused avoidance based on the WHO (Reference 5) Well-Being index (1998) which measure the absence of a positive effect and has been validated for use in children.

Yoga may be an effective approach for reducing emotional distress and promoting pro-social behavior in youth (Yoshihara, Hiramoto, Oka, Kubo, \& Sudo, 2014) and has implications for the prevention of psychosomatic symptoms in healthy women. Somatization is defined as

\section{“A tendency to experience and communicate somatic distress in response to psychosocial stress and to seek medical help for it."}

In addition, according to Yoshihara et al., it costs the United States' health care system an estimated over $\$ 100$ billion annually (Slovacek, Tucker, \& Pantoja, 2003). Medically unexplained physical symptoms can include: headache, dizziness, chest pain, lower back pain, and nausea, muscle soreness, breathing problems, hot or cold spells, and numbness or tingling in parts of the body, among others. There are numerous treatments for somatization and they have varying degrees of effectiveness (Yoshihara, Hiramoto, Oka, Kubo, \& Sudo, 2014). Findings suggest that yoga can improve somatization and mental health status (Sharma, Singhal, Singh, \& Sharma, 2013).

In a study regarding headaches (migraine), yoga intervention was found significant in reducing the headache activity, medication intake, symptoms, anxiety and depression in migraine without aura (Libera, Colombo, Pavan, \& Comi, 2014). Further studies are required to investigate safety and efficacy of CAM in pediatric headache, as a possible adjunctive treatment/medication to conventional pharmacological approach (es). Promising for the utilization of yoga, youth aged 10-17, who suffer from headaches, are 2.1 times more likely to use CAM compared with youth who do not experience 
headache. CAM is often not used as a direct remedy, but for better stress management which can be a relevant fact in headache. Kuttner et al. reported that stress and anxiety can exacerbate IBS symptoms and that yoga holds promise as an intervention for adolescents with IBS.

In a study of obese breast cancer survivors, Littman et al, concluded that there were no statistically significant differences in quality of life and fatigue among women in a yoga group compared to women in the control group, however, these two measures did improve. Again, as with most studies to date, more studies are needed to pursue the application of yoga for various physical issues. (Littman, et al., 2010) And to also explore the possible inference to children.

\section{METHODS}

A seventeen-item questionnaire was developed to assess changes in well-being for children in fourth or fifth grade associated with participating in a weekly youth yoga program for eight weeks. Responses for all seventeen outcome questions (dependent variables) used a Likert Scale with five response categories (i.e., always, often, sometimes, rarely, never). The individual questions were developed by the administration of a non-profit youth yoga organization which advocates for the mental and emotional health benefits of practicing yoga. As children suffer from an increasingly greater amount of stress and anxiety, the non-profit youth yoga organization created a nonexperimental, pilot research study as a way to obtain feedback from the children they serve. The survey that the yoga organization formulated was to ascertain levels of anxiety, depression, somatization, hyperactivity, and personal relationships by creating survey questions that were age and grade level appropriate from January - March, 2014. Well-being concepts were measured based upon observations yoga teachers made based upon previous yoga classes they taught for the youth yoga organization. These observations were based upon personal experience and are subjective. They are not based upon objective, empirical data. The intervention was the practice of yoga which occurred twice per week for sixty minutes for eight weeks. Each yoga class was followed by a twenty-minute nutrition and environmental health lesson.

There was no control group in this study as the yoga organization at the time was not interested in obtaining feedback from children who do not participate in its yoga program. The two independent variables that were measured in the study were time (pre- and post-survey) and grade (either $4^{\text {th }}$ or $5^{\text {th }}$ grade). Time was the primary predictor variable for all analyses. Five dependent variables; which are measures of mental, emotional, and social well-being were analyzed to ascertain change over time. The five independent variables are: self-esteem, interpersonal relationships, somatization, anxiety and depression.

\section{RESEARCH DESIGN}

The study design is non-experimental as there was no control group and no random assignment to the intervention group. The goal of the study was to identify and observe changes over time related to yoga. This study employed the use of a survey as a program evaluation tool to assess participant improvement in measures of mental well-being over time and after participating in the yoga pilot program.

\section{Data Collection}

This survey was administered on the first and last day of the yoga intervention with assistance from the yoga teacher. Subjects were recruited from schools that agreed to have the eight-week yoga program in school. Students voluntarily agreed to participate in the yoga program and complete the evaluation survey. All students were allowed to participate who were in the fourth or fifth grade at specific schools. Participants were then asked to attend a weekly yoga program for eight weeks which lasted approximately one hour each week. Participants completed a baseline survey and an eight-week follow-up survey which took them 10-15 minutes to complete depending on their reading ability and assistance needed.

\section{Materials USed}

The program evaluation survey was developed by yoga teachers and organizational administrators who were seeking feedback from the children on the yoga program. The youth yoga organization was also interested in how the yoga program improved various dimensions of well-being. The valuation questionnaire was not based upon scientific literature and was not tested for reliability or validity. 
The survey was formulated as a result of the opinions and ideas from experts in the field of yoga for children.

\section{Measures}

Measures were developed for this study by the youth yoga organization that provided the yoga program in schools. Questions were developed from previous meetings of yoga teachers and the yoga organization administration. Seventeen questions were written for program evaluation by the youth yoga organization. All students voluntarily participated in the evaluation process both before the program started and also at the end of the program during the final class session. The measures that were selected for this study were selected because they were most strongly associated with both the physical and mental health measures of interest by the investigators.

The only predictor or independent variables obtained by the youth yoga organization were grade level (fourth or fifth grade) and time point (pre/post yoga program). Five survey questions were selected for analysis because they could be more closely correlated with scientific literature than other questions.

The first measure selected is, "I like the person I am," which was developed as a measure of selfworth and self-esteem. Members of the youth yoga organization believe that yoga was an activity that taught each child that he or she has inherent value and abilities. These talents and attributes could be identified easier when children are physically active, calm, focused, and relaxed which are direct benefits of yoga practice.

The second measure, "It's easy for me to make friends," measures the level of confidence students have in themselves which, in turn, is beneficial in forming relationships with other students. The yoga program administrators and teachers believeif students become more calm and relaxed by practicing yoga, they will be able to form friendships easier and feel better about themselves.

The third measures, "I get headaches," can be a measure of somatization. Many students reported experiencing some type of discomfort in their face or head. The youth yoga organization believed this could be attributed to either dehydration or stress. Yoga is an activity that promotes self-care (drinking plenty of water) and decreases stress.

The fourth measure, "I have a hard time sitting still," was formed to assess concentration and mindfulness. Children have reported having difficulty paying attention, sitting still, focusing, and maintaining control of their bodies (including breathing) in classrooms. The youth yoga organization believed yoga would be an activity that could help students focus and concentrate in class. Yoga was also an activity that could be practiced to help students alleviate the feeling of excess energy such as fidgeting and movements. Students would be taught deep, slow, and controlled breathing techniques that they could use when they felt nervous or distracted which would help alleviate these physical and mental symptoms.

The fifth measure is, "I get stomachaches or feel sick." The yoga organization felt that kids may have some digestion issues and complaints about pain or a sick feeling in the "stomach" or gastrointestinal (GI) tract. Yoga has for many years suggested that specific poses are helpful aids to digestion and the entire GI system.

\section{ANALYSIS}

Data were analyzed using SPSS 22.0®. Descriptive (i.e., frequencies, percentages, means, and standard deviations) analyses were conducted for seventeen outcome variables of which five are reported in these findings. Statistical analysis of the results included Wilcoxon Signed-Rank Test for pre- and post-intervention comparisons. All assumptions for using the Wilcoxon Signed-Rank Test were met: 1) Ordinal variable (Likert Scale); 2) same subjects in both groups; and, 3) Distribution between the two related groups was symmetrical in shape. Descriptive statistics were conducted for the two independent variables (i.e., time and grade) and five outcome measures (i.e., mental wellbeing measures). Inferential analyses included non-parametric tests; specifically, the Wilcoxon Signed-Rank Test to measure changes over time (i.e., pre-post) for the five measures (i.e., ordinal variables). 
Note: Grade was not a significant predictor or covariate for any of the outcome measures of mental well-being, thus it was not included in the results reporting.

\section{EThical Considerations}

Study approval was obtained from a regional accredited university's IRB determined this study to be exempt because secondary data analysis was conducted. No identifying information was collected by the yoga organization that collected the data. The only known information about the students are their grade and responses on the evaluation survey regarding well-being. Students were assigned a number as a way to link the pre-survey with the post-survey. The file that had students' names and numbers was maintained separately from the surveys and survey data. This file has subsequently been destroyed to ensure confidentiality and anonymity.

\section{RESUlTS}

Table1. Frequencies of selected responses among 4th and 5th grade students who participated in an eight-week yoga program for a five-item Likert Scale survey in San Diego County, California.

\begin{tabular}{|c|c|c|c|c|c|c|c|}
\hline Outcome & Time & $\begin{array}{c}\text { Always } \\
\mathrm{n}(\%)\end{array}$ & $\begin{array}{l}\text { Often } \\
\mathrm{n}(\%)\end{array}$ & $\begin{array}{c}\text { Sometimes } \\
\mathrm{n}(\%)\end{array}$ & $\begin{array}{c}\text { Rarely } \\
\mathrm{n}(\%)\end{array}$ & $\begin{array}{l}\text { Never } \\
\mathrm{n}(\%)\end{array}$ & $\begin{array}{c}\text { Total } \\
(\mathrm{n})\end{array}$ \\
\hline \multirow{2}{*}{$\begin{array}{l}\text { I like the person I } \\
\text { am }\end{array}$} & pre & $\begin{array}{c}25 \\
(43 \%)\end{array}$ & $13(22 \%)$ & $\begin{array}{c}15 \\
(26 \%)\end{array}$ & $\begin{array}{c}5 \\
(9 \%)\end{array}$ & 0 & 58 \\
\hline & post & $35(60 \%)$ & $15(26 \%)$ & $\begin{array}{c}8 \\
(14 \%) \\
\end{array}$ & 0 & 0 & 58 \\
\hline \multirow{2}{*}{$\begin{array}{l}\text { It is easy for me to } \\
\text { make new friends }\end{array}$} & pre & $18(31 \%)$ & $15(25 \%)$ & $\begin{array}{c}15 \\
(25 \%)\end{array}$ & $\begin{array}{c}5 \\
(9 \%)\end{array}$ & $\begin{array}{c}5 \\
(9 \%)\end{array}$ & 58 \\
\hline & post & $18(31 \%)$ & $25(43 \%)$ & $\begin{array}{c}12 \\
(21 \%)\end{array}$ & $\begin{array}{c}2 \\
(3 \%)\end{array}$ & $\begin{array}{c}1 \\
(2 \%)\end{array}$ & 58 \\
\hline \multirow{2}{*}{ I get headaches } & pre & $18(31 \%)$ & $18(31 \%)$ & $\begin{array}{c}7 \\
(12 \%)\end{array}$ & $10(17 \%)$ & $\begin{array}{c}5 \\
(9 \%)\end{array}$ & 58 \\
\hline & post & $13(22 \%)$ & $17(29 \%)$ & $\begin{array}{c}22 \\
(38 \%) \\
\end{array}$ & $\begin{array}{c}5 \\
(9 \%) \\
\end{array}$ & $\begin{array}{c}1 \\
(2 \%)\end{array}$ & 58 \\
\hline \multirow{2}{*}{$\begin{array}{c}\text { It is hard for me to } \\
\text { sit still }\end{array}$} & pre & $13(22 \%)$ & $11(19 \%)$ & $\begin{array}{c}6 \\
(10 \%) \\
\end{array}$ & $13(22 \%)$ & $15(26 \%)$ & 58 \\
\hline & post & $17(29 \%)$ & $18(31 \%)$ & $\begin{array}{c}11 \\
(19 \%)\end{array}$ & $\begin{array}{c}8 \\
(14 \%) \\
\end{array}$ & $\begin{array}{c}4 \\
(7 \%) \\
\end{array}$ & 58 \\
\hline \multirow{2}{*}{$\begin{array}{l}\text { I get stomach aches } \\
\text { or feel sick }\end{array}$} & pre & $\begin{array}{c}7 \\
(12 \%) \\
\end{array}$ & $18(31 \%)$ & $\begin{array}{c}21 \\
(36 \%)\end{array}$ & $10(17 \%)$ & $\begin{array}{c}2 \\
(3 \%)\end{array}$ & 58 \\
\hline & post & $16(28 \%)$ & $21(36 \%)$ & $\begin{array}{c}18 \\
(31 \%)\end{array}$ & $\begin{array}{c}3 \\
(5 \%) \\
\end{array}$ & 0 & 58 \\
\hline
\end{tabular}

Table one represents the outcomes, pre- and post-survey, for fifty-eight 4th and 5th grade students who participated in an eight-week yoga intervention. This table showed the descriptive analysis of the student's difference in responses to five survey questions before and after the intervention.

I like the person I am (self esteem) - The number of student who answered "Always" and "Often" increased from $43 \%(n=25)$ to $60 \%(n=35)$ and $22 \%(n=13)$ to $26 \%(n=15)$, respectively. This was as expected.

It is easy for me to make new friends (interpersonal relationships) - For the positive response of "Always," the expected increase did not occur, but remained the same as the pre-yoga survey results. However, for the positive response of "Often," an increase was observed from $25 \%$ ( $\mathrm{n}=15$ ) to $43 \%$ $(\mathrm{n}=25)$ while results for "Never" and "Rarely" decreased; also a positive effect.

I get headaches (somatization) - As expected there was a decrease in the number of respondents who reported "Always" and "Often" from $31 \%(n=18)$ to $22 \%(n=13)$ and $31 \%(n=18)$ to $29 \%(n=17)$, respectively. There was a shift, post yoga intervention, to the "Sometimes" group $12 \%$ ( $\mathrm{n}=7$ ) to $38 \%$ $(n=22)$.

It is hard for me to sit still (anxiety and depression) - There was an unexpected increase in responses of "Always" and "Often" from 22\% (13) to 29\% (17) and 19\% (n=11) to $31 \%(\mathrm{n}=18)$.

I get stomach aches or feel sick (somatization) - This was also an unexpected increase for responses of "Always" and "Often" from $12 \%(n=7)$ to $28 \%(n=16)$ and $31 \%(n=18)$ to $36 \%(n=21)$. 
Table2. Pre-and post-yoga intervention comparisons of well-being measures among 4th and 5th grade students who participated in an eight-week yoga program in San Diego County, California (1-5 scale with 5 being the highest and 1 being the lowest)

\begin{tabular}{|c|c|c|c|c|c|}
\hline Outcome & Time & Mean & SD & $\mathrm{Z}$ & $\mathrm{p}$ \\
\hline \multirow{2}{*}{ I like the person I am } & pre & 4.00 & 1.03 & \multirow{2}{*}{-3.42} & 0.001 \\
\hline & post & 4.47 & 0.73 & & \\
\hline \multirow{2}{*}{ It is easy for me to make new friends } & pre & 3.62 & 1.25 & \multirow{2}{*}{-2.30} & 0.020 \\
\hline & post & 3.97 & 0.92 & & \\
\hline \multirow{2}{*}{ I get headaches } & pre & 3.21 & 1.14 & \multirow{2}{*}{-2.36} & 0.018 \\
\hline & post & 3.60 & 0.97 & & \\
\hline \multirow{2}{*}{ It is hard for me to sit still } & pre & 2.90 & 1.54 & \multirow{2}{*}{-3.46} & 0.001 \\
\hline & post & 3.62 & 1.24 & & \\
\hline \multirow{2}{*}{ I get stomachaches or feel sick } & pre & 3.31 & 1.01 & \multirow{2}{*}{-3.08} & 0.001 \\
\hline & post & 3.83 & 0.90 & & \\
\hline
\end{tabular}

All five outcomes significantly increased over time according to the non-parametric test performed on the ordinal variables. First, "I like the person that I am," the mean significantly increased $(p<0.01)$ from $4.00(\mathrm{SD}=1.03)$ to $4.47(\mathrm{SD}=0.73)$; "It is easy for me to make new friends" significantly increased $(\mathrm{p}<0.01)$ from $3.62(\mathrm{SD}=1.25)$ to $3.97(\mathrm{SD}=3.97)$; "I get headaches" significantly increased $(\mathrm{p}<0.01)$ from $3.21(\mathrm{SD}=1.14)$ to $3.60(\mathrm{SD}=0.97)$; "It is hard for me to sit still" significantly increased $(\mathrm{p}<0.01)$ from $2.90(\mathrm{SD}=1.54)$ to $3.62(1.24)$; and, "I get stomach aches or feel sick" significantly increased $(\mathrm{p}<0.01)$ from $3.31(\mathrm{SD}=1.01)$ to $3.83(\mathrm{SD}=0.90)$.

\section{DISCUSSION}

\section{I like the person I am}

Significant findings in this study suggest three positive outcomes and two unfortunate side effect outcomes. First, students showed they significantly liked themselves better over time in this program. Results show the student's perception of liking the person they are improved after the yoga program. This may indicate that yoga can positively improve children's self-esteem. Research has shown the yoga is related with higher levels of self-esteem and self-worth (Eggleston, Middlestadt, Lindeman, McCormick, \& Koceja, 2011).

\section{It's easy for me to make new friends}

For the second outcome variable, "It is easy for me to make new friends," results showed that students believed they could more easily begin new friendships after completing the yoga program. Yoga may be positively related to factors associated with interpersonal relationships.

\section{I get headaches}

For the outcome variable, "I get headaches," findings suggested that students reported a decrease in headaches after the yoga program. Headache can be a symptom of stress and tension from psychological and social challenges in a child's daily life. Yoga can reduce stress for many people and is one of the more common reported benefits (Eggleston, et. al., 2011).

\section{It's hard for me to sit still}

The results did not show a logical outcome of practicing yoga. Students may have misunderstood the question as the expected result would have been for children to report that it is easier to sit still. Children may have been asked the final survey before yoga started on the last day of yoga class and they may have been excited to participate and thus had a higher level of difficulty at that time period, but not overall. Perhaps a new question asking about concentration or paying attention may have been a better for this measure.

\section{I get stomachaches or feel sick}

The results of this survey question had an unexpected outcome in that the responses did not increase favorably. Students may have misunderstood the question. The yoga intervention may have been done when the children were hungry or after lunch when they had just eaten. The yoga program is generally offered in the early afternoon or late morning. There are no reported cases in the literature supporting or disproving the relationship of yoga with stomachaches or feeling sick, specifically.

In responding to survey questions, it is possible that the students answered in a way which they believed was desirable instead of what they thought was the best answer. Students may have not answered honestly or did not understand the questions that they were asked. Test and re-test is a threat to validity in that the children may not have answered the question appropriately which indicated a change and introduces recall bias. 
In adults with mental illness, it is usually apparent in childhood and adolescence. Teaching children to be mindful and present and to have an internal locus of control early in life can be beneficial to well-being. Children have reported in other studies that they develop the ability to self-regulate their emotions.

Results of the study are limited by the research design (non-experimental) because there was no control group and random assignment was not made or possible. No causation can be inferred from the results as there are potential confounding variables that could explain some of the significant findings. Thus, the best conclusion can be made regarding this study is that there is an association of yoga with all five well-being outcomes which were analyzed.

Yoga programs in schools provide a possible solution to the increased prevalence of psychological, social, and emotional health issues for children including stress, anxiety, depression, attention deficit disorders, and bullying. Scientific measures must be developed or modified so that they accurately reflect positive changes to these psychosocial-emotional issues. The youth yoga organization's results show some promise that children may be able to improve their self-worth, self-esteem, and ability to get along well with other students. Also, physical health may improve because stress is decreased in children after practicing yoga which leads to optimal physiological conditions.

\section{Future Research}

The next step for yoga research is to develop new objective measures addressing mental, social, and emotional health well-being. These measures should be based upon a thorough review of scientific literature on well-being for children and also for measures that other researchers have used when looking at the benefits of yoga for children. One suggestion would be to use the Positive And Negative Affect Scale (PANAS) which is a 10-item scale that measures positive and negative mood states/emotions/attitude (Watson, Clark\& Tellegen, 1988). An improved research design can also be achieved in this study by the addition of a control group of students who do not practice yoga from the same school where yoga is being taught. The children practicing yoga should be the same demographically and also in terms of health and well-being. Ideally, the only difference between these two groups would be the yoga program. If possible, randomization could be used to randomly assign participants to either a yoga or control group. This would allow the youth yoga organization to have an experimental study for the evaluation of their yoga program and experimental design is considered the gold standard in research.

Control groups, and group random assignment, experimental studies address more threats to internal validity than non-experimental studies. By having greater internal validity, an experimental study will have the best chance of determining whether or not the yoga intervention had a causal effect on the treatment group. Furthermore, any findings from an experimental study can be applied to the population from which the study's samples were drawn.

In the future there should be a control group and the study should be more experimental in design. Validity and reliability could be improved by using indicators of mental and physical well-being indicated in scientific literature. The organization should use valid and reliable scientific measures for well-being in elementary-age students. A larger sample size would increase statistical power. Adding independent variables such as age, gender, and socioeconomic status should be added.

\section{REFERENCES}

[1] An, B., Avshalon, S., \& Ehud, M. (2010). Here and now: yoga in Israeli schools. International Journal of Yoga, 42

[2] Elwy, A., Groessl, E., Eisen, S., Riley, K., Maiya, M., Lee, J., . . Park, C. (2014). A systematic scoping review of yoga intervention components and study quality. American Journal of Preventative Medicine, 220-232.

[3] Frank, J., Bose, B. A., \& Zelmer, V. D. (2012). Transformative life skills and training program curriculum. Oakland: Niroga Institute.

[4] Frank, J., Bose, B., \& Schrobenhauser-Clonan, A. (2014). Effectiveness of a school-based yoga program on adolescent mental health, stress coping strategies, and attitudes toward violence: findings from a high-risk sampe. Journal of Applied School Psychology, 29-49.

[5] Galatino, M., Galbavy, R., \& Quinn, L. (2008). Therapeutic effects of yoga for children: a systematic review of the literature. Pediatric Physical Therapy, 66-80.

[6] Get the facts: yoga for health. (2013, June). Retrieved from National Institutes of Health U.S. Department of Health and Human Services: http:/www.NIH.gov 
[7] Geyer, R., Lyons, A., Amazeen, L., Alishio, L., \& Cooks, L. (2011). Feasibility study: the effect of therapeutic yoga on quality of life in children hospitalized with cancer. Pediatric Physical Therapy, 375-379.

[8] Harper, J. (2010). Teaching Yoga in Urban Elementary Schools. International Journal of Yoga Therapy, 99-109.

[9] Harper, J. (2010). Yoga Therapy in Practice. Teaching yoga in urban elementary schools, 99109.

[10] Jensen, P., \& Kenny, D. (2004). The effects of yoga on the attention and behavior of boys with Attention-Deficit/hyperactivity disorder (ADHD). Journal of Attention Disorders, 205-216.

[11] Kaley-Isley, L., Peterson, J., Fischer, C., \& Peterson, E. (2010). Yoga as a complimentary therapy for children and adolescents: a guide for clinicians. Psychiatry, 20-32.

[12] Khalsa, S., Hickey-Schultz, L., Cohen, D., Steiner, N., \& Cope, S. (2012). Evaluation of the mental health benefits of yoga in a secondary school: a preliminary randomized controlled trial. The Journal of Behavioral Health Services and Research, 80-90.

[13] Khalsa, S., Shorter, S., Cope, S., Wyshak, G., \& Sklar, E. (2009). Yoga ameliorates performance anxity and mood disturbance in young professional musicians. Applied Psychophysical biofeedback, 279-289.

[14] Kuttner, L., Chambers, C., Hardial, J., Israel, D., Jacobson, K., \& Evans, K. (2006). A randomized trial of yoga for adolescents with irritable bowel syndrome. Pain Research and Management, 217.

[15] Libera, D., Colombo, B., Pavan, G., \& Comi, G. (2014). Complementary and alternative medicine (CAM) use in an Italian cohort of pediatric headache patients: the tip of the iceberg. Neurological Science, 145-148.

[16] Littman, A., Bertram, L., Ceballos, R., Ulrich, C., Ramaprasad, J., McGregor, B., \& McTeirnan, A. (2010). Randomized controlled pilot trial of yoga in overweight and obese breast cancer survivors: effects on quality of life and anthropometric measures.

[17] Menon, B., Satyanand, V., \& Karishma, P. (2013). Effects of yoga on tension headache. Journal of Dr. NTR University of Health Sciences, 167.

[18] Nidhi, R., Padmalatha, V., Nagarathna, R., \& Amritanshu, R. (2012). Effect of yoga program on quality of life in adolescent polycystic ovarian syndrome: a randomized control trial. Bangalore: Springer.

[19] Santangelo, L. (2012). Reducing stress in school-age girls through mindful yoga. Journal of Pediatric Health Care, 45-56.

[20] Shapiro, D., Cook, I., Davydov, D., Ottaviani, C., \& Leuchter, A. M. (2007). Yoga as a complementary treatment of depression: effects of traits and moods on treatment outcomes. eCAM, 493-502.

[21] Sharma, N., Singhal, S., Singh, A., \& Sharma, C. (2013). Effectiveness of integrated yoga therapy in treatment of chronic migraine: randomized controlled trial. The Journal of Headache and Pain, 116.

[22] Skowronek, I., Mounsey, A., Handler, L., \& Guthmann, R. (2014). Can yoga reduce the symptoms of anxiety and depression. The Journal of Family Practice, 398-399, 407.

[23] Slovacek, S., Tucker, S., \& Pantoja, L. (2003). A study of the yoga ed program at the accelerated school. Los Angeles: PERC.

[24] Telles, S., Singh, N., Bhardawaj, A., Kumar, A., \& Balkrishna, A. (2013). Effect of yoga or physical exercise on physical, cognitive and emotional measures in children: a randomized controlled trial. Child \& Adolescent Psychiatry \& Mental Health, 7:37.

[25] Well-being concepts. (2015, February 3). Retrieved from Centers for Disease Control and Prevention: http://www.cdc.gov/hrqol/wellbeing.htm

[26] archeski, T., Mahon, N., Yarcheski, A., \& Hanks, M. (2010). Perceived Stress and Wellness in Early Adolescents Using the Neuman Systems Model. The Journal of School Nursing, 230-237.

[27] Yoshihara, K., Hiramoto, T., Oka, T., Kubo, C., \& Sudo, N. (2014). Effect of 12 weeks of yoga training on the somatization, psychological symptoms, and stree-related biomarkers of health women. BioPsychoSocial Medicine, 1-9. 